\title{
Effect of an ectoparasite on lay date, nest-site choice, desertion, and hatching success in the great tit (Parus major)
}

Anne Oppliger

Heinz Richner

Philippe Christe

Zoology Department,

University of Bern,

CH-3032 Hinterkappelen,

Switzerland
A. Oppliger and P. Christe are also at the Zoology Department, University of Lausanne, $\mathrm{CH}-1015$ Lausanne, Switzerland.

Received 9 October 1992

Revised 2 February 1993

Accepted 8 March 1999

$1045-2249 / 94 / \$ 5.00$

- 1994 International Society for Behavioral Ecology

Ectoparasites are common in most bird species, but experimental evidence of their effects on life-history traits is scarce. We investigated experimentally the effects of the hematophagous hen flea (Ceratophyllus gallinae) on timing of reproduction, nest-site choice, nest desertion, clutch size, and hatching success in the great tit (Parus major). When great tits were offered a choice on their territory between an infested and a parasite-free nest-box, they chose the one without parasites. When there was no choice, the great tits in a territory containing an infested nest-box delayed laying the clutch by 11 days as compared with the birds that were offered a parasite-free nesting opportunity. The finding that there was no difference in phenotypic traits related to dominance between the birds nesting in infested boxes and birds nesting in parasite-free boxes suggests that the delay is not imposed by social dominance. Nest desertion between laying and shortly after hatching was significantly higher in infested nests. There was no difference between infested and parasite-free nests in clutch size, but hatching success and hence brood size at hatching were significantly smaller in infested nests. Nest-box studies of great tits have been seminal in the development of evolutionary, ecological, and behavioral theory, but recently a polemic has arisen in the literature about the validity of the conclusions drawn from nest-box studies where the naturally occurring, detrimental ectoparasites are eliminated by the routine removal of old nests between breeding seasons. Our study suggests that this criticism is valid and that the evaluation of the effects of ectoparasites may improve our understanding of behavioral traits, life-history traits, or population dynamics. Key words: ectoparasites, great tit, hatching success, life-history traits, nest desertion, Parus major, timing of breeding. (Behav Ecol $5: 130-134(1994)]$

$\mathrm{T}$ iming of reproduction is an important life-history trait in most organisms, and in particular in species in temperate areas with strongly seasonal reproduction. Many studies, using the natural variation in laying date, demonstrated a correlation between the timing of reproduction and reproductive success of birds (e.g., Daan et al., 1990; Perrins, 1965, 1970). In an elegant experimental study, Verhulst and Tinbergen (1991) showed that the timing of reproduction per se influences reproductive success in the great tit (Parus major), and hence they demonstrated the causal relationship between the life-history trait and reproductive success. For an understanding of the evolution of this trait, it is thus important to identify the factors that influence a bird's decision to start reproduction.

Several studies on great tits have demonstrated a behavioral response of the timing of breeding to annual fluctuation in environmental conditions. In great tits the laying date (Dhondt and Eyckerman, 1979; Kluijver, 1951, 1952; Perrins and McCleery, 1989; Van Balen, 1978) correlates with spring temperature: in cold springs laying can be delayed by several weeks. Because spring temperatures are well correlated with growth of leaves and their associated herbivorous caterpillars, which are the main prey of great tits during breeding, Perrins and McCleery (1989) suggested that spring temperatures affect date of laying mainly via its direct effect on food supply. In an experimental study, Källan- der (1974) showed that females supplied with extra food a few weeks before breeding initiated laying 4-5 days earlier than unfed females. Further, Dhondt (1989) showed that middle-aged females advance laying by 2 days compared with first-year females and 3 days compared to 5-year-old females.

The numerous nest-box studies of great tits have been seminal in the development of evolutionary, ecological, and behavioral theory. However, Moller (1989) questioned the generality of the conclusions drawn from nest-box studies and pointed out that the results of these studies may be affected by two artifacts: the design of nest-boxes may drastically reduce predation, and the routine removal of old nests between breeding seasons eliminates naturally occurring, detrimental ectoparasites.

Our studies on the great tit (Christe et al., in press; Richner et al., 1993) aim to elucidate experimentally the effects of an ectoparasite, the hematophagous hen flea (Ceratophyllus gallinae), on important life-history traits of their hosts. The hen flea is a highly common ectoparasite of great tits and of other hole-nesting birds (Harper et al., 1992). Hundreds of fleas survive in the nest inside their cocoon to the next breeding season and emerge early in spring (Bates, 1962; Humphries, 1968). Hen fleas have detrimental effects on the reproductuve success of their hosts (Richner et al., 1993), and from this it can be expected that the birds take this ectoparasite into account when deciding when and where they will start to breed. Here we report 
the effects of the hen flea on nest-box choice, nest desertion, laying date, hatching success, and hatchling number.

\section{METHODS}

\section{Host population}

We carried out the study during the breeding seasons of 1991 and 1992 in a forest surrounding the campus of the University of Lausanne, Switzerland. The forest is mainly composed of beech trees, interspersed with a few oaks and hombeam. There is little undergrowth except at the edge of the forest. A population of great tits has been breeding there in nest-boxes since 1989. Adult birds were color-ringed as nestlings or during mist-netting in winter.

For each nest we recorded laying date, hatching date, clutch size, and brood size at hatching. Laying date was recorded as the day the first egg was laid. A nest was considered deserted if it was abandoned between laying of the first egg and hatching and, in a second comparison, if abandoned between laying of the first egg and the fourth day after hatching. When the chicks were 14 days old, we captured both parents and measured their body mass, tarsus and wing lengths, and determined their age as either first year or older by the color of the primary covers.

Statistical analysis was performed using the Systat Statistical Package (Wilkinson, 1989). Percentage values and proportions were arcsine-transformed for parametric statistical tests. All significance values reported are two-tailed unless indicated otherwise.

\section{Ectoparasites}

The ectoparasite used in this study was the naturally occurring hen flea (Ceratophyllus gallinae). The hen flea is very common in great tit nests. In 1989, in the new nest-boxes and without any manipulation of fleas, more than $80 \%$ of all nests were naturally infested with the flea. The hen flea normally produces two generations per nest (Harper et al., 1992). In a natural situation the second generation starts to emerge just a few days before fledging (Oppliger et al., personal observations). Many fleas leave with the nestlings, dozens of fleas at a time wait at the entrance hole for visitors, and hundreds remain inside the nest in cocoons, attached to the nesting material until the start of the following breeding season. In the forest studied here, the predominant ectoparasite is the hen flea; hematophagous mites were absent during both experimental years, and two boxes contained blowflies, which we removed during regular nest inspections.

We removed the tit nests the day of fledging in both 1990 and 1991. Each nest was cut in half. One half was kept in a plastic bag and its fleas used for later infestations, and the other half was used for other purposes. In the experiments described below, nests were either infested with fleas, or the fleas were eliminated by a heat treatment of the nests. For the latter purpose we took the nests out of the nest-boxes, put them in a sealed plastic bag to prevent loss of humidity, and heated the nests for 5 minutes inside a microwave oven. This pro-
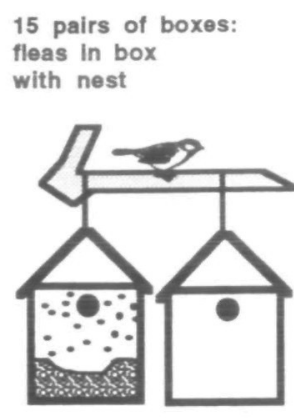

box chosen for nesting:
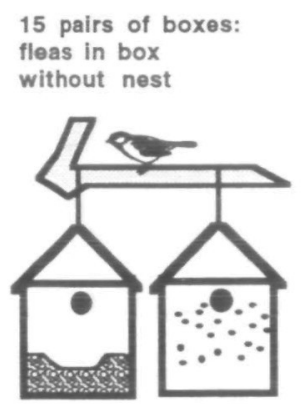

9
2
15 pairs of boxes: no fleas

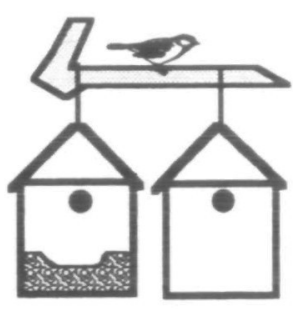

8

5 cedure was carried out in the field using a transportable 220-volt generator to power the microwave.

\section{Experiment I}

We designed the first experiment to test whether great tits prefer for nesting (1) a clean nest-box over a box containing a nest, and (2) a clean nestbox over a box infested with the hematophagous flea. After the breeding season of 1990 , we provided each of 45 territories of great tits a pair of nest-boxes, placed beside each other at a distance of $0.3-1 \mathrm{~m}$ (see Figure 1 for the experimental design). One box of each pair contained an old, parasite-free nest. In the middle of the next winter, in 30 of these pairs of boxes, 1 box was infested with approximately 20 fleas. In 15 of these 30 pairs of boxes the fleas were injected in the box with the nest and in the other 15 pairs they were put in the clean box. We repeated this treatment around 20 March. The third group of 15 pairs of nest-boxes was kept free of parasites.

\section{Experiment 2}

The purpose of the second experiment was to test whether birds in an infested nest-box without a choice of nest-boxes in their territory would delay breeding. We therefore removed, after the end of the breeding season of 1991 , one of the boxes of a pair described in the first experiment, leaving only one nest-box per territory. To increase sample size we included nine more territories in our study plot, which makes a sample of 54 nest-boxes. A randomly chosen sample of 25 nest-boxes was then furnished with one-half of a heat-treated old nest. Another sample of 29 nest-boxes was provided with one-half of an infested nest. We repeated the heat-treatment in the middle of March 1992, before the start of the breeding season. Of the 25 parasite-free boxes, 23 were taken by great tits and 2 boxes by other species. One female was found dead in the box after having laid 5 eggs (data accepted for laying date but excluded from other analyses), and for another female laying date is not exactly known. Of the 29 infested boxes, 20 were taken by great tits, 3 by other species, and 6 boxes stayed empty to the end of the season. Our basic sample size for laying date is therefore 22 parasite-free boxes and 20 infested boxes; for clutch size, 22 parasite-free and 16 infested broods ( 4 females abandoned during laying); and for hatching date, hatching success, and hatchling number 21 parasite-free broods (one
Figure 1

Design and results of the choice experiment. A pair of nest-boxes was provided in each of 45 territories. One box of each pair contained an old nest, and in $\mathbf{3 0}$ of these pairs hematophagous ectoparasites were injected in either the box with or without the nest. 
Table 1

Desertion between laying the first egg and hatching in infested and parasite-free nest-bores

\begin{tabular}{llc} 
& Birds & \\
\cline { 3 - 3 } Fleas & Stayed & Deserted \\
\hline Absent & 21 & 1 \\
Present & 13 & 7 \\
\hline
\end{tabular}

female abandoned during incubation) and 13 infested broods ( 3 females abandoned during incubation). Hatching success was calculated as the number of chicks hatched divided by clutch size. It is given for the undeserted nests, and also for all nests, including the nests that were deserted after laying.

\section{RESULTS}

\section{Experiment 1}

In the 45 available pairs of adjacent boxes, 36 pairs of great tits built nests and laid eggs in one box of the pair, and 9 pairs of boxes were not used by great tits. In none of the pairs of boxes were the two boxes simultaneously occupied. There was no preference (binomial test, $p=.62$ ) for the boxes containing an old nest (Figure 1). Overall, 56\% of the great tits started breeding in the box containing the nest.

In contrast to the indifference toward the presence or absence of a nest, the great tits clearly preferred the parasite-free box of a pair (Figure 1). Of the 23 pairs of great tits that started breeding in the 30 experimental pairs of boxes, $78 \%$ of birds chose the parasite-free box (binomial test, $p=.01$ ).

\section{Experiment 2}

In parasite-free nests, only one out of 22 clutches was abandoned between laying the first egg and hatching the chicks, whereas in infested nests 7 out of 20 nests were abandoned during the same time hematophagous ectoparasite, the hen flea.
Table 2

Desertion between laying the first egg and day 4 after hatching in infested and parasite-free nest-boxes

\begin{tabular}{lcc} 
& \multicolumn{2}{c}{ Birds } \\
\cline { 2 - 3 } Fleas & Stayed & Deserted \\
\hline Absent & 21 & 1 \\
Present & 8 & 12 \\
\hline
\end{tabular}

period (Table 1; Fisher's Exact test, $p=.018$ ). No further desertion between hatching and day 4 after hatching occurred in parasite-free nests, but five more infested nests were abandoned, raising desertion to 12 out of 20 nests in infested nest-boxes (Table 2; Fisher's Exact test, $p<.001$ ).

The birds in the infested nest-boxes started their clutch on average 11 days later than the ones in the parasite-free boxes (Table 3 and Figure 2). Hatching of the chicks in the infested boxes was delayed by 10 days, and the difference between infested and parasite-free broods for laying date and hatching date is highly significant (Table 3).

Clutch size was not significantly affected (Table 3) by the ectoparasite factor, but the number of hatchlings was clearly reduced in the presence of ectoparasites. Considering nondeserted broods only, there were 1.7 fewer hatchlings in the infested nests (Table 3). The difference is significant and arose by a difference in hatching success. Taking all broods, the infested nests contained 3.1 fewer hatchlings (Table 3 ) than the parasite-free broods.

Hatching success of the clutches that were not abandoned during incubation, calculated as the ratio of the number of hatchlings on the number of eggs, was $88 \%$ in the parasite-free broods, but $69 \%$ only in infested broods. Hatching success is significantly lower in the infested broods as shown by a statistical comparison of the arcsine-transformed ratios (Table 8 ). Hatching success in parasite-free broods was not influenced by hatching date, but decreased in the infested broods with hatching date (Figure 3). This interaction between hatching date and the experimental factors (i.e., the parasite manipulation) is significant (ANCOVA, $F_{1,30}=6.68$, $p=.015$ ).

\section{DISCUSSION}

Our study demonstrates that great tits in infested nest-boxes, compared with birds in clean boxes, delay egg laying by 11 days. For great tits it has been shown that survival of juveniles is negatively correlated with hatching date (Dhondt and Olaerts, 1981; Kluijver et al., 1977; Perrins, 1965). Using a data set covering 25 years, Perrins and McCleery (1989) showed for Whytham (Oxford, England) that the number of recruits per brood decreases on average by $3.7 \%$ for each day's delay in starting to lay. A difference of II days would then cause a reduction in reproductive success of $40 \%$. Why should a bird at a parasitized nesting site delay breeding?

The fleas in a nest-box survive in large numbers from one breeding season to the next. Hundreds to thousands of flea larvae are present when the fledglings leave the nest. Only few adult fleas leave the nest with the fledglings (Humphries, 1968). The 
Table 3

Breeding parameters (means \pm SD) of infested and parasite-free nests

\begin{tabular}{lclllll}
$\begin{array}{l}\text { Nest } \\
\text { treatment }\end{array}$ & Laying date & Clutch size & $\begin{array}{l}\text { Hatching } \\
\text { date }\end{array}$ & $\begin{array}{l}\text { Hatching } \\
\text { success }\end{array}$ & $\begin{array}{l}\text { Brood size } \\
\text { at hatching }\end{array}$ & $\begin{array}{l}\text { Overall production } \\
\text { of hatchlings }\end{array}$ \\
\hline Infested & $\mathbf{3 0 . 4 \pm 1 2 . 1}$ & $\mathbf{8 . 4} \pm 1.6$ & $51.9 \pm 11.8$ & $1.05 \pm .38$ & $5.8 \pm 2.5$ & $4.0 \pm 9.4$ \\
Parasite-free & $19.2 \pm 10.5$ & $8.4 \pm 1.4$ & $41.7 \pm 10.1$ & $1.29 \pm .27$ & $7.4 \pm 2.0$ & $7.1 \pm 2.6$ \\
$t$ (sample size) & $\mathbf{3 . 2 ( 2 0 , 2 2 )}$ & $0.1(16,22)$ & $2.7(13,21)$ & $2.2(13,21)$ & $2.1(13,21)$ & $U=104.5(20,22)$ \\
$p$ & .003 & .95 & .011 & .037 & .044 & .003 \\
\hline
\end{tabular}

Brood size at hatching excludes nests that were abandoned between laying and hatching. Overall production of hatchlings includes nests that were abandoned between laying the first egg and hatching. Hatching success is given as the arcsinetransfonned proportions of brood size at hatching over clutch size.

- Day 1 = 1 April.

'Sample sizes of infested and parasite-free nests, respectively.

flea larvae in the nest develop into adults after chicks fledge, but then stay in a dormant stage insides cocoons, mostly attached to the nesting material. As described by Humphries (1968), mechanical stimulation of the old nest, as naturally provided by a nesting bird, causes the fleas to emerge from the cocoon. Similarly, a change in temperature causes emergence. In spring, the emerged fleas wait around the entrance hole for visiting birds, often forming a black ring, but also emigrate by crawling out of the nest-box and up the tree trunk (Humphries, 1968). If the nest-box is not occupied, fewer and fewer fleas will therefore be left in time in the box, and finally the flea load will be so small that the site becomes acceptable for a bird intending to breed. Nevertheless, breeding later has costs (Perrins and McCleery, 1989), and a bird will therefore have to trade off the reduction in reproductive success caused by the delay in breeding against the loss it would incur if breeding earlier with a higher load of ectoparasites. We cannot, however, on the basis of our data, argue that the observed delay in laying date is a strategic decision, given the fact that hatching success decreases (Figure 3) with laying date in infested boxes. For this to be true it would have to be shown experimentally that birds in infested boxes that delay date of laying enjoy higher reproductive success than birds in infested boxes that do not delay their breeding attempt. The benefits from a delay of 11 days would have to be higher than the costs imposed by a lower hatching success.

It could be argued that the observed delay of laying date in our study was caused by a difference in quality or dominance of birds nesting in parasitefree or in infested nest-boxes. That is, higher-quality birds would take the parasite-free boxes early in the season, leaving the parasitized ones to the birds of lower quality. Gamett (1981) suggested that body size (tarsus) can affect dominance behavior in great tits. A comparison of tarsus length and body mass of both males and females breeding in infested and parasite-free boxes shows no difference (Table 4), and we also found no difference in wing length or age between birds in infested and parasite-free boxes. Behavioral observations in the territories also indicate that there was no change of territory owners related to the experimental distribution of infested and parasite-free boxes. It is therefore unlikely that the delay is caused by factors related to phenotypic differences in breeding birds.

The observation that hatching success in infested broods was reduced by nearly $20 \%$ is important because it leads to a significantly lower brood size at hatching as shown here. Møller (1990) has also shown a significant reduction (98.4\% versus $91.1 \%)$ in hatching success of barn swallows (Hirundo rustica) affected by the tropical fowl mite (Ornithonyssus bursa). We cannot at present offer a sound interpretation of our finding. Nilsson and Smith (1988) found that female blue tits that were experimentally fed during incubation tended to have higher hatching success than unfed females, although the difference in that study was only $6 \%$ (98\% versus $92 \%$ ). Lyon and Montgomerie (1985) prevented male snow buntings (Plectrophenax nivalis) from feeding their mates during incubation and also found a decrease in hatching success. Thus, energetic limitations during incubation, when energy reserves are thought to be lowest in the great tit (Yom-Tov and Hilborn, 1981), seem to affect hatching success. In our study a more severe effect on energy reserves of the females in infested broods could arise either directly by the action of the fleas that suck blood from the incubating female or by the fleas affecting the male's pattern of incubation feeding and hence the female's energy reserves.

\section{Figure 3}

Regression of hatching success on hatching date in parasite-free nests $(O)$ and in nests infested $(\bullet)$ with a hematophagous ectoparasite. Some data points have identical values.

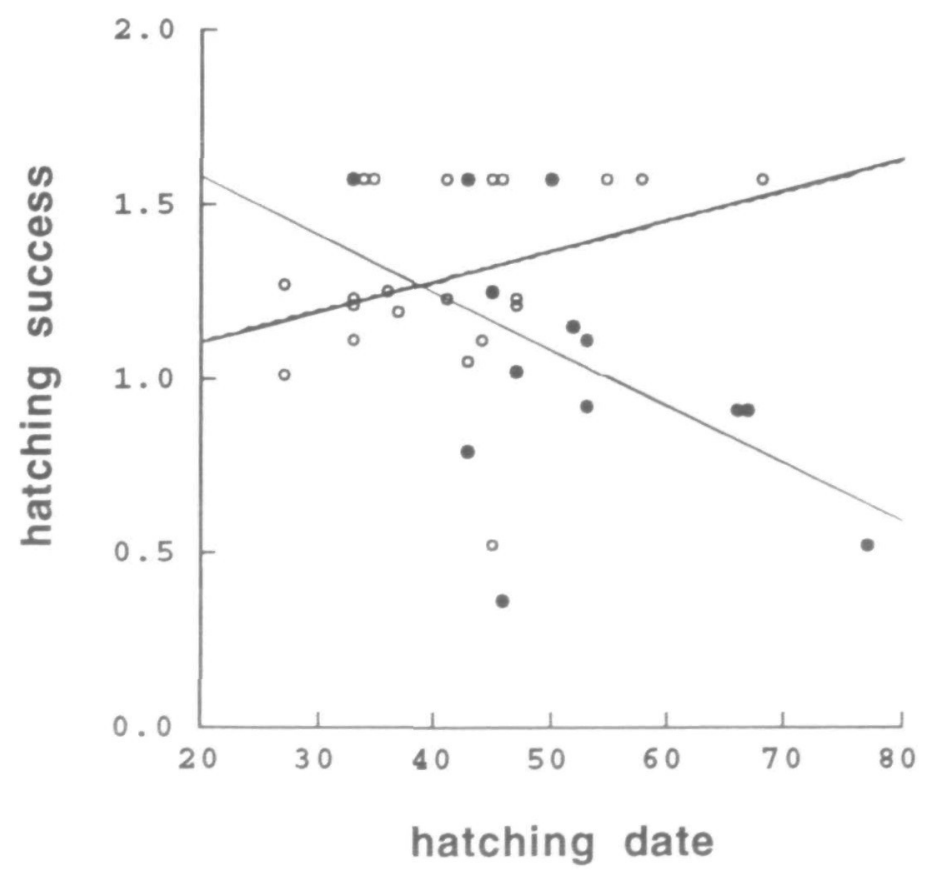




\begin{tabular}{llllll} 
& Males & & Females & \\
\cline { 2 - 3 } \cline { 5 - 6 } Nest treatment & Body mass & $\begin{array}{l}\text { Tarsus length } \\
(\mathrm{mm})\end{array}$ & & $\begin{array}{l}\text { Tarsus length } \\
\text { Body mass }\end{array}$ & $\begin{array}{l}\text { (mm) } \\
\text { Infested }\end{array}$ \\
Parasite-free & $17.8 \pm 0.9$ & $23.1 \pm 1.0$ & & $17.4 \pm 0.8$ & $22.1 \pm 0.6$ \\
\hline
\end{tabular}

None of the differences between infested and parasite-free nests is significant $(\phi>.1)$.

However, other hypotheses explaining the decrease of hatching success in infested broods cannot be excluded. Alternative causes are currently being studied.

Recently a polemic has arisen about the validity of nest-box studies as a research tool (Koenig et al., 1992; Møller, 1989, 1992). Møller (1989) pointed out that the load of detrimental ectoparasites may be considerably reduced in nest-box studies by the annual cleaning procedure of boxes by the researchers, and hence the effects of such parasites on nest choice, mate choice, reproductive success, and nestling growth have been ignored in most studies. Our study supports Møller's (1989) contention that these effects should not be ignored. Our first experiment demonstrates the effects of hen fleas on choice of nest sites, and their effects on reproduction, excluding the time effect, have been documented elsewhere (Richner et al., 1993). The sccond experiment shows the effect of fleas on laying date and hatching success. The timing of reproduction is a life-history trait that strongly affects reproductive succcss, and a great effort has been spent in the past to understand the factors that affect laying date in the great tit and other birds. We believe that an understanding of the evolution of this trait requires detailed knowledge of the effects of ectoparasites on the trait. Like Moller (1992), we do not, with these remarks, seek to invalidate any of the excellent long-term studies on the great tit and other bird species, but wish to draw attention to the need to incorporate the effects of ectoparasites on demographic, behavioral, and cvolutionary traits in future studies.

We thank Manfred Milinski, Jost $M$. Tinbergen, and an anonymous referee for their valuable comments. We gratefully acknowledge support by the Swiss National Science Foundation, grants 31-26606.89 and 81-27217.89, to H. Richner.

\section{REFERENCES}

Bates JK, 1962. Field studies on the behaviour of bird fleas. I. Behaviour of the adults of three species of bird fleas in the field. Parasitology 52:113-192.

Christe P, Oppliger A, Richner H, in press. Ectoparasite affects choice and use of roost sites in the great tit (Panus major). Anim Behav.

Daan S, Dijkstra C, Tinbergen JM, 1990. Family planning in the kestrel (Falco tinnunculus): the ultimate control of covariation of laying date and clutch size. Behaviour 114:83-116.

Dhondt AA, 1989. The effect of old age on the reproduction of the great tits Parus major and blue tits Parus caeruletus. Ibis 181:268-280.

Dhondt AA, Eyckerman R, 1979. Temperature and date of laying by tits Parus ssp. Ibis 121:329-331.

Dhondt AA, Olaerts G, 1981. Variations in survival and dispersal with ringing date as shown by recoveries of Belgian great tits Parus major. Ibis 123:96-98.

Crarnett MC, 1981. Body size, its heritability and influence on juvenile survival among grcat tits, Parus major. Ibis 123:31-41.

Harper GH, Marchant A, Boddington DG, 1992. The ecology of the hen flea Ceratophyllus gallinae and the moorhen flea Dasypsyllus gallinulae in nest boxes. J Anim Ecol 61:317-327.

Humphries DA, 1968. The host-finding behaviour of the hen flea, Ceratophyllus gallinae (Schrank). Parasitology 58:403-414.

Källander H, 1974. Advancement of laying of great tits by the provision of food. Ibis 116:365-367.

Kluijver HN, 1951. Population ecology of the great tit Parus m.major. Ardea 39:1-135.

Kluijver HN, 1952. Notes on body weight and time of breeding in the great tit Parus m.major. Ardea 40:123141.

Kluijver HN, Van Balen JH, Cave AJ, 1977. The occurrence of time saving mechanisms in the breeding biology of the great tit, Parus major. In: Evolutionary ecology (Stonehouse B, Perrins CM, eds). London: Macmillan; 153-169.

Koenig WD, Gowaty PA, Dickinson JL, 1992. Boxes, barns, and bridges: confounding factors or exceptional opportunities in ecological studies? Oikos 63:305-308.

Lyon BE, Montgomerie RD, 1985. Incubation feeding in snow buntings: female manipulation or indirect male parental care. Behav Ecol Sociobiol 17:279-284.

Møller AP, 1989. Parasites, predators and nest boxes: facts and artefacts in nest box studies of birds? Oikos $56: 421-423$.

Meller AP, 1990. Effects of parasitism by a haematophageous mite on reproduction in the barn swallow. Ecology 71:2345-2357.

Møller AP, 1992. Nest boxes and the scientific rigour of experimental studies. Oikos 63:309-311.

Nilsson J-Ä, Smith HG, 1988. Incubation feeding as a male tactic for early hatching. Anim Behav 36:641647.

Perrins CM, 1965. Population fluctuations and clutch size in the great tit, Parus major. J Anim Ecol 34:601-647.

Perrins CM, 1970. The timing of birds' breeding seasons. Ibis 1 12:242-255.

Perrins CM, McCleery RH, 1989. Laying dates and clutch size in the great tit. Wilson Bull 101:236-253.

Richner H, Oppliger A, Christc P, 1993. Effect of an cctoparasite on reproduction in grcat tits. J Anim Ecol 62:703-710.

Van Balen JH, 1973. A comparative study of the breeding ecology of the great tit Parus major in different habitats. Ardea 61:1-93.

Verhulst S, Tinbergen JM, 1991. Experimental evidence for a causal relationship between uming and success of reproduction in the great tit Parus m. major. J Anim Ecol 60:269-282.

Wilkinson L, 1989. SYSTAT: the system for statistics. Evanston, Illinois: SYSTAT.

Yom-Tov Y, Hilborn R, 1981. Energetic constraints on clutch size and time of breeding in temperate zone birds. Oecologia 48:234-243. 